\title{
Efectos del aula virtual como estrategia de enseñanza- aprendizaje en el logro de aprendizajes significativos en estudiantes universitarios
}

\author{
Effects of the virtual classroom as a teaching-learning \\ strategy in the achievement of significant learning in \\ university students
}

\author{
Damiana Flores Mamani*
}

\begin{abstract}
Resumen
Con el paso de los años se va tomando mayor interés por la calidad educativa y la tecnología de información y comunicación ( TIC) va tomando fuerza a nivel mundial; es por eso que, la investigación se orientó a determinar los efectos del aula virtual como estrategia de enseñanza - aprendizaje en el logro de aprendizajes significativos en estudiantes universitarios, esta investigación es de enfoque cuantitativo, de tipo experimental y de diseño preexperimental con prueba de entrada y prueba de salida. Se trabajó con 15 estudiantes matriculados .Los resultados de la investigación demuestran que la aplicación del aula virtual tiene efectos positivos en los estudiantes para el logro de sus aprendizajes significativos, para establecer la escala de valoración del nivel de logro de los aprendizajes se utilizó la rúbrica; dando como resultado que de un total de 15 estudiantes, 10 que representa el $67 \%$ lograron una escala de valoración de excelente con promedios entre 17 a 20 puntos. En conclusión, los efectos del aula virtual en estudiantes universitarios desarrollan el conocimiento y el rendimiento académico de manera planificada y organizada con contenidos tecnológicos, en un espacio intersubjetivo con una meta en común centrada en la construcción social del conocimiento a través de actividades de aprendizaje centradas más en el estudiante.
\end{abstract}

Palabras clave: Aprendizaje, aula virtual, enseñanza, estrategia, enseñanza virtual.

\begin{abstract}
As the years go by, there is more interest in educational quality and information and communication technology (ICT) is gaining strength worldwide; that is why the research was oriented to determine the effects of the virtual classroom as a teaching-learning strategy in the achievement of significant learning in university students, this research is of quantitative approach, experimental type and pre-experimental design with entrance test and exit test. The results of the research show that the application of the virtual classroom has positive effects on the students for the achievement of their significant learning, to establish the evaluation scale of the level of achievement of the learning, the rubric was used; resulting in a total of 15 students, 10, representing 67\%, achieved a rating scale of excellent with averages between 17 to 20 points. In conclusion, the effects of the virtual classroom on university students develop knowledge and academic performance in a planned and organized manner with technological contents, in an intersubjective space with a common goal focused on the social construction of knowledge through learning activities centered more on the student.
\end{abstract}

Keywords: Learning, virtual classroom, teaching, strategy, virtual teaching.

\section{Introducción}

Enla última década ha habido una inclinación por aumentar la calidad de la educación (Aldana de Becerra, 2008), por la escasez de nuevas formas de enseñanza y una manera de conseguirlo y evidenciarlo es lograr formar en las aulas profesionales con competencias para investigar, con mayor pertinencia social y capaces de generar conocimiento que le apunten a la solución de problemas en el ámbito profesional a nivel local, regional y nacional (Levison \& Salguero, 2009) ,la formación investigativa en pregrado actualmente es deficiente, en aspectos relacionados con los procesos actuales de enseñanza y aprendizaje de la investigación debido a algunos obstáculos que impiden alcanzar una adecuada formación

* Universidad Nacional del Altiplano Puno, Perú. 0000-0003-0062-3286, e-mail: damianaflores@unap.edu.pe 
en este campo (Aldana de Becerra,2012). Según Betancur (2010), la mayor causa que se ha encontrado para el poco desarrollo $y$ estimulación de los estudiantes para realizar investigación en la Educación Superior es por la forma como el docente desarrolla las clases, las cuales, en la mayoría de los casos, se centran en presentaciones magistrales. Lo anterior, da poco espacio a la interpretación de la información, a la reflexión crítica, preguntar, al problematizar situaciones, crear soluciones a la problemática de su entorno.

Históricamente han existido diferentes posiciones frente a la relación existente entre el desarrollo de cada individuo y su aprendizaje, Vygotsky(1978a), en un intento por armonizarlas, expone que el aprendizaje es parte importante del desarrollo y asegura que sobre él influye directamente el grado de maduración del sistema nervioso; por esta razón los profesores necesitan conocer la teoría de la mente, con el fin de identificar la necesidad de enseñar y averiguar qué es lo que deben enseñar, para adaptarlo al nivel de habilidad del alumno (Kline,2015),el desarrollo de habilidades, ante todo la transferencia del aprendizaje, o sea, lograr que el aprendizaje anterior, sobre todo el aprendizaje metodológico, sirva para el aprendizaje presente y que éste último potencie aprendizajes futuros (Ausubel, 1983). Son también fundamentales las habilidades de flexibilidad, adaptabilidad e interdisciplinariedad o manejo de problemas desde distintos puntos de vista disciplinarios. Por otra parte, González y colaboradores, definen el aprendizaje como un acto complejo que demanda participación, actitud crítica, reflexión y búsqueda activa de información.

Hoy en día las plataformas educativas virtuales han tomado una gran significación en la enseñanza- aprendizaje de los estudiantes, estas tecnologías de información y comunicación (TIC) están tomando fortaleza a nivel mundial. Según Lozano (2004), utiliza el término "triángulo del E-Learning” para relacionar la tecnología, los contenidos y los servicios. El autor se refiere a la tecnología (plataformas online, campus virtuales, etc.) como elemento básico que se ha convertido en un comodity, que ha pasado de venderse a unos precios astronómicos a encontrarse hoy con acceso libre y gratuito, a los contenidos como cursos estándares y cursos a medida que son cada vez más demandados y que cada día son más económicos, y a los servicios como la acción de tutores, profesores online, documentación en la biblioteca virtual, y todo un abanico de posibilidades desde el chat a la conexiones online, que ayuda a desarrollar los múltiples modelos del E-Learning; pero no obstante Rosenberg (2001), lo define como el uso de tecnologías Internet para la entrega de un amplio rango de soluciones que mejoran el conocimiento y el rendimiento. Está basado en tres criterios fundamentales: El E-Learning trabaja en red, lo que lo hace capaz de ser instantáneamente actualizado, almacenado, recuperado, distribuido, y permitiendo compartir instrucción o información, es entregado al usuario final a través del uso de ordenadores utilizando tecnología estándar de Internet y se enfoca en la visión más amplia del aprendizaje que van más allá de los paradigmas tradicionales de capacitación. Autores como Esquivel, I. (2013. p. 252), manifiesta: “...la Educación online ha mostrado mayor efectividad rompiendo las barreras del tiempo y espacio, al ofrecer métodos, técnicas y recursos que hacen más efectivo y flexible el proceso enseñanza - aprendizaje, a través del uso de tecnologías como la radio, la televisión, el vídeo, los sistemas de informática y software interactivos...”, sin embargo, es necesario tener presente que la actual disponibilidad y rapidez de acceso a las grandes cantidades de información impacta significativamente en el esquema social, así como en sus prioridades y preferencias. La educación como eje fundamental de la sociedad y la cultura no puede ser ajena a estos cambios y se ha visto obligada a renovar sus escenarios y estrategias, cambiando el rol que tradicionalmente ocupan docentes y estudiantes (Falco.2017), reenfocándose en la resolución de problemas y usando las TIC como herramienta para enriquecer la didáctica, no solo capturando la atención de los estudiantes y divirtiéndose, sino también resignificando el aula con contenidos contextualizados que los inviten a leer y a transformar su realidad próxima (Castro,2005), utilizandola motivación ylaintención de aprender de los estudiantes como estimulantes de su propio desarrollo (Vygotsky,1978b) y considerando las diferencias en los presaberes, las personalidades y los estilos de aprendizaje de los estudiantes (Cohen y Baruth,2017).Entre los recursos TIC, que a partir de su integración con la pedagogía 
conllevan a aprendizajes significativos, resaltan las aulas virtuales (Basantes et al.,2017), las cuales ayudan el aprendizaje autónomo, propio de la corriente pedagógica constructivista, (EstebanAlbert y Zapata-Ros,2016), a la vez que amplían la cobertura del curso y facilitan la construcción de actividades interactivas ajustadas a los intereses del grupo objetivo, así como la inserción de contenidos multimedia existentes y el registro sistematizado de los procesos individuales. De igual forma el uso de herramientas informáticas incentiva a las nuevas generaciones a utilizar las TIC con propósitos educativos.

Ahora bien, en consonancia con la masificación del uso de las TICs para la enseñanza en general, y la popularización del uso de las mismas con el fin de facilitar el aprendizaje de una segunda lengua, desde hace un par de décadas atrás algunos autores han comenzado a reflexionar en torno al call (Computer assisted language learning), así como sobre su incidencia en el aprendizaje de lenguas. Tal es el caso de Last; Hubbard; Warschauer y Healy; y Chapelle, autores que en los últimos años han realizado diferentes aportes a este campo desde su conceptualización, hasta sugerencias acerca de su futuro y orientación en general. Mientras este proceso ocurre en la virtualidad, las aulas de clase se han concentrado más en resaltar las habilidades particulares de cada aprendiz y en buscar la manera de potenciar las mismas mediante procesos metacognitivos. Por esta razón se buscaron los aportes más relevantes a cada una de estas visiones, así como los posibles intentos de integrar ambas tendencias de manera exitosa. La investigación se orientó a determinar los efectos del aula virtual como estrategia de enseñanza- aprendizaje en estudiantes universitarios de la UNA Puno, durante el año académico 2019.

\section{Materiales y método}

Se llevó a cabo la investigación en la Escuela Profesional de Educación Primaria en de la Facultad de Ciencias de la Educación, de la Universidad Nacional del Altiplano, ubicada en el Distrito, Provincia y Departamento de Puno, a 3827 msnm a orillas del lago Titicaca, el lago navegable más alto del mundo; Puno se encuentra localizado en la sierra del sudeste del país en la meseta del Callao a: $13^{\circ} 0066^{\prime} 00^{\prime}$ y $17^{\circ} 17^{\prime} 30^{\prime \prime}$ de latitud sur y los $71^{\circ} 06^{\prime} 57^{\prime \prime}$ y $68^{\circ} 48^{\prime} 46^{\prime \prime}$ de longitud oeste del meridiano de Greenwich. Limita por el Sur, con la región Tacna. Por el Este, con la República de Bolivia y por el Oeste, con las regiones de Cusco, Arequipa y Moquegua. El clima de la ciudad es lluvioso durante los meses de septiembre a abril y frígido en la estación de invierno, debido a la altitud y latitud en la que se encuentra.

El estudio realizado se da en estudiantes de las zonas Rurales de los diferentes Provincias y Distritos de la Región, con niveles de inteligencia emocional y tipos de personalidad, teniendo características de introvertidos y en algunos casos tímidos lo cual ha permitido llevar este trabajo de investigación.

a) El diseño utilizado fue el muestreo, la población y muestra no se asignaron al azar, es decir ya existía al pre experimento al respecto la población de investigación está constituida por los estudiantes que cursaron el año académico 2019 de la Escuela Profesional de Educación Primaria, la población y muestra está constituida por los estudiantes del IV semestre (15 estudiantes matriculados) de la Universidad Nacional del Altiplano de Puno.

b) Descripción de materiales, se utilizó la rúbrica para poder medir el nivel de logro de los aprendizajes. Es necesario reivindicar ese potencial formativo y rescatar las posibilidades que la rúbrica ofrece para orientar a los estudiantes hacia qué se espera de ellos, cómo hacer un buen trabajo, qué es lo más relevante y, por tanto, dónde hay que poner el énfasis (Blanco, 2008). En esta investigación se trabajó con dos variables, la variable independiente que es efectos de efectos del aula virtual como estrategia de enseñanza - aprendizaje y la variable dependiente aprendizaje significativo.

c) La aplicación de la prueba estadística con la cual se trabajó fue la T de student, siendo de tipo experimental, con diseño pre -experimental, con prueba de entrada y salida, en un solo grupo. Además para los datos cuantitativos se aplicaron normas estadísticas. 


\section{Resultados y discusión}

Las sociedades actuales son lo que son por el creciente protagonismo tecnológico que existe, sin embargo, no debe perderse de vista que las TIC no son la solución a todos los problemas y que son herramientas que nos permiten explotar diferentes rutas y acciones por experimentar, sobre todo en el ámbito educativo, donde el foco de atención está en los retos del desarrollo de habilidades y destrezas que son susceptibles de computarizar como la creatividad o la inteligencia social (Cobo, 2016).

Los resultados obtenidos en el pre test nos da a conocer que los estudiantes no se encuentran en la escala valorativa de (17 a 20), de acuerdo a la teoría sustentada por Vallés (1997),al no aplicarse estrategias adecuadas para el logro de aprendizajes no va permitir en el individuo el conjunto de operaciones enteramente organizadas para procesar una información .Por lo anterior los recursos TIC, que a partir de su integración con la pedagogía conllevan a aprendizajes significativos, resaltan las aulas virtuales (Basantes et al.,2017), las cuales ayudan el aprendizaje autónomo, propio de la corriente pedagógica constructivista, (Esteban-Albert y Zapata-Ros,2016),a la vez que amplían la cobertura del curso y facilitan la construcción de actividades interactivas ajustadas a los intereses del grupo objetivo, así como la inserción de contenidos multimedia existentes y el registro sistematizado de los procesos individuales.

Tabla 1. Resultados de la escala de valoración de la prueba de entrada.

\begin{tabular}{|c|c|c|}
\hline $\begin{array}{c}\text { ESCALA DE } \\
\text { VALORACIÓN }\end{array}$ & $\begin{array}{c}\mathrm{N}^{\circ} \mathrm{DE} \\
\text { ESTUDIANTES }\end{array}$ & $\%$ \\
\hline Excelente (17 a 20) & 0 & 0 \\
\hline Bueno (14 a 16) & 5 & 33 \\
\hline Regular (11 a 13) & 7 & 47 \\
\hline Malo (00-10) & 3 & 20 \\
\hline TOTAL & 15 & 100 \\
\hline
\end{tabular}

La tabla 1 demuestra que en un $0 \%$ de los estudiantes no se encuentran en la escala de valoración de excelente con los promedios de (17 a 20) puntos y en un 47\% están en la escala de valoración de regular con los promedios de (11 a
13), estos resultados indican que su aprendizaje debe mejorarse para lograr aprendizajes más significativos y duraderos.

Tabla 2. Resultados de la aplicación del aula virtual como estrategia de enseñanzaaprendizaje en estudiantes universitarios.

\begin{tabular}{lcc}
\hline \multicolumn{1}{c}{$\begin{array}{c}\text { ESCALA DE } \\
\text { VALORACIÓN }\end{array}$} & $\begin{array}{c}\mathbf{N}^{\circ} \mathbf{D E} \\
\text { ESTUDIANTES }\end{array}$ & $\%$ \\
\hline Excelente (17 a 20) & 6 & 40 \\
Bueno (14 a 16) & 5 & 33 \\
Regular (11 a 13) & 3 & 20 \\
Malo (00-10) & 1 & 7 \\
TOTAL & 15 & 100 \\
\hline Funte: Rúbrica de Evaluación -2019. &
\end{tabular}

Fuente: Rúbrica de Evaluación - 2019.

La tabla 2 demuestra que en un 1\% de los estudiantes no se encuentran en la escala de valoración de, bueno y regular y en $40 \%$ se encuentra en la escala de valoración de excelente con promedios de (17 a 20), esta escala es significativa lo que indica que la aplicación del aula virtual como estrategia de enseñanzaaprendizaje tiene efectos positivos en los estudiantes.A decir de Mominó y Sigáles (2016) en el ámbito educativo es donde debiera hacerse más evidente el conjunto de transformaciones tecnológicas-sociales puesto que en este contexto es donde se producen y socializan los seres humanos. Sin embargo, la promesa de las tecnologías informáticas en red dista mucho de haberse cumplido con la amplitud prevista. Lo anterior, debido a que entre las habilidades tecnológicas de los llamados nativos digitales se interpone la práctica educativa obsoleta, los principios organizativos, las culturas obsoletas de la enseñanza y las dificultades inherentes a la creación de nuevos aprendizajes (Mominó y Sigáles, 2016).

Tabla3. Resultados de la escala de valoración de la prueba de salida.

\begin{tabular}{lcc}
\hline \multicolumn{1}{c}{$\begin{array}{c}\text { ESCALA DE } \\
\text { VALORACIÓN }\end{array}$} & $\begin{array}{c}\mathbf{N}^{\circ} \text { DE } \\
\text { ESTUDIANTES }\end{array}$ & \% \\
\hline Excelente (17 a 20) & 10 & 67 \\
Bueno (14 a 16) & 4 & 26 \\
Regular (11 a 13) & 1 & 7 \\
Malo (00-10) & 0 & 0 \\
TOTAL & 15 & 100 \\
\hline
\end{tabular}

Fuente: Prueba de salida - 2019. 
La tabla 3 demuestra que en un $67 \%$ de los estudiantes se encuentran en la escala de valoración de excelente con los promedios de (17 a 20) puntos y en un 0\% están en la escala de valoración de malo con los promedios de (00 a 10), estos resultados nos indica que los estudiantes universitarios lograron aprendizajes significativos (difundir información existente ,desarrollar capacidades necesarias para el aprendizaje permanente, actualización del conocimiento y habilidades necesarias que le ayudaran en su vida profesional). Autores como Mayer (2002) plantean ideas como: que el aprendizaje es personal e individual, cada uno construye sus propios significados; a ritmos diferentes y por medios distintos a lo largo de etapas similares de aprendizajes específicos; algo activo e integrativo.

\section{Conclusiones}

Al determinar los efectos del aula virtual como estrategia de enseñanza-aprendizaje en estudiantes universitarios se muestra que el aprendizaje logrado es significativo; del total de 15 estudiantes, 10 que representa el $67 \%$ lograron una escala de valoración de excelente con promedios entre (17 a 20) puntos. Se realizó la prueba de hipótesis, donde se acepta la hipótesis alterna ( $5>1.4$ ), esto nos indica que los efectos de las aulas virtuales como estrategia de enseñanza -aprendizaje en estudiantes universitarios desarrolla el conocimiento y el rendimiento académico de manera planificada y organizada con contenidos tecnológicos, en un espacio intersubjetivo con una meta en común centrada en la construcción social del conocimiento a través de actividades de aprendizaje centrados más en el estudiante.

\section{Agradecimientos}

A todas aquellas personas e instituciones que me apoyaron en la investigación.

\section{Referencias bibliográficas}

Aldana de Becerra, G. M. (2008), Enseñanza de la investigación y epistemología de los docentes Educación y Educadores [en línea] 2008.

Aldana de Becerra, G. M. (2012). La formación investigativa: su pertinencia en pregrado.
Revista Virtual Universidad Católica del Norte, 1(35), 367-379. Recuperado de: http://revistavirtual.ucn.edu.co/index.php/ RevistaUCN/article/view/366/681

Ausubel, D. Psicología Educativa: Un Punto de Vista Cognoscitivo. México: Trillas, 1983.

Basantes, A.V., M.E. Naranjo, M.C. Gallegos y N.M. Benítez, Los Dispositivos Móviles en el Proceso de Aprendizaje de la Facultad de Educación Ciencia y Tecnología de la Universidad Técnica del Norte de Ecuador, doi: $\quad 10.4067 / S 0718-50062017000200009$, Formación Universitaria, 10, 79-88 (2017)

Betancur, M.R. (2010). La actitud estudiantil sobre la investigación en la universidad. Un estudio de caso en seis universidades de Colombia. Investigación \& Desarrollo, 18(2). Recuperado de: http://search.proquest.com/openview/ ff6951543283d31e8c5104aaa89a2a25/1? pqorigsite $=$ gscho

Blanco, A. (2008). Las rúbricas: un instrumento útil para la evaluación de competencias. En L. Prieto (Coord.). La enseñanza universitaria centrada en el prendizaje (171188). Barcelona: Octaedro/ICE Universidad de Barcelona.

Cobo, C. (2016). La Innovación Pendiente. Reflexiones (y provocaciones) sobre educación, tecnología y conocimiento. Montevideo: Colección Fundación Ceibal.

Castro, M. (2005). La Ciencia es una Forma Cultural Necesaria para Vivir, Entrevista a Neus Sanmartí, Especialista en Didáctica de las Ciencias

Cohen, A. y O. (2017). Baruth, Personality, Learning, and Satisfaction in Fully Online Academic Courses, doi: 10.1016/j. chb.2017.02.030, Computers in Human Behavior

Chapelle, Carol. Computer Applications in Second Language Acquisition: Foundations for Teaching, Testing, and Research. London: Cambridge University Presss, 2001. Impreso. doi: 10.1017/CBO9781139524681

EsquivelI. (2013). Estado del conocimiento sobre la educación mediada por ambientes virtuales de aprendizaje: Una aproximación a través de la producción de tesis de grado y posgrado. Revista Mexicana de Investigación Educativa, Vol.18, No.56, p.249-264

Esteban-Albert, M. y M. Zapata-Ros. (2016). Estrategias de Aprendizaje y eLearning. Un 
Apunte para la Fundamentación del Diseño Educativo en los Entornos Virtuales de Aprendizaje, doi: http://dx.doi.org/10.6018/ red/50/15, RED, Revista de Educación a Distancia

Hubbard, Philli. "Elements of call methodology: development, evaluation, andimplementation”. The Power of call (1996): 15-32. Impreso

Falco, M. (2017) Reconsiderando las Prácticas Educativas: TICs en el Proceso de EnseñanzaAprendizaje, doi: 10.15366/tp2017.29.002, Tendencias Pedagógicas, 29, 59-76

Kline, M. A. (2015).How to Learn About Teaching : An Evolutionary Framework for the Study of Teaching Behavior in Humans and Other Animals, doi:10.1017/ S0140525X140000901-71, Behavioral and Brain Sciences, 1-71

Hernández Sampieri, R., Fernández Collado, C. y Baptista Lucio, P. (2003) Metodología de la investigación (3a ed.). México: McGraw-Hill.

Last, Rex W. Artificial Intelligence Techniques in Language Learning. Chichester: Ellis Horwood, 1989. Impreso.

Levison, Y. C. O., \& Salguero, L. A. (2009). Una propuesta de competencias investigativas para los docentes universitarios. Recuperado de: $\quad$ http://www.redalyc.org/articulo. oa?id=76120651006
Lozano, J. (2004). El triángulo del E-learning. Zona interactiva. Recuperado de http:// www.telepolis.com/cgi-bin/web/ DISTRITODOCVIEW? url $=\mid 1589 / \mathrm{doc} /$ Reflexiones/triangulo.htm

Mayer, Richard. (2002). Psicología de la Educación. El aprendizaje en las Áreas del Conocimiento. Madrid: Pearson Educación, S.A.

Mominó, J. M., \& Sigáles, C. (2016). El impacto de las TIC en la educación. Más allá de las promesas. Barcelona: UOC Ediciones.

Rosenberg, M. (2001). E-Learning: Strategies for Delivering Knowledge in the Digital Age. Editorial McGraw Hill, 2000, pág.344.

Santibáñez J. (2010). Aula virtual y presencial en aprendizaje de comunicación audiovisual y educación. Comunicar: Revista Científica Iberoamericana De Comunicación Y Educación, (35), 183-191. ISSN 1134-3478.

Vallés, A. A. (1997). Aprender a aprender. Edit CIEP. Lima Perú.

Vygotsky, L.S., Interaction Between Learning and Development, Mind and Society, doi: 10.1016/ S0006-3495(96)79572-3, 29-36 (1978a)

Vygotsky, L.S., Tool and Symbol in Child Development Chapter, Mind and Society (1978b)

Warschauer, Mark y Deborah Healy. "Computers and language learning: an overview”. Language Teaching 31 (1998): 51-71. 